\title{
On destabilizing effects of two fundamental non-conservative forces
}

\author{
R. Krechetnikov*, J.E. Marsden \\ Control and Dynamical Systems MS 107-81, California Institute of Technology, Pasadena, CA 91125, United States
}

Received 1 June 2005; received in revised form 15 November 2005; accepted 9 December 2005

Communicated by J. Lega

\begin{abstract}
In this work we discuss the instabilities in mechanical systems caused by two fundamentally different non-conservative forces, referred to as dissipative and positional forces, each of which may lead to energy dissipation. One of the objectives of this discussion is to recall and to put into the context of current research some of the important classical results by Thomson-Tait-Chetayev and Merkin, which are under-appreciated nowadays: many new examples, e.g. radiation in Hamiltonian systems, the Levitron, etc., appearing in recent literature can be interpreted with the help of these classical results. Next, in the spirit of the Lagrange-Dirichlet theory, we introduce the geometric picture of the phase space corresponding to the effects of destabilization in finite-dimensional systems. On the physical side, our objective is to demonstrate that both of these types of non-conservative forces appear quite commonly and often simultaneously in physical systems. As an illustration, we consider the Levitron, a system in which the dissipative effects have not heretofore been studied. Finally, using Nikolai's elastic bar model as a paradigm, we discuss the notion of a secondary dissipation-induced instability, when the above two fundamental non-conservative forces interact. This unified way of thinking should help us to understand the intricate links among various mechanical systems through the most fundamental mechanisms in their behavior.
\end{abstract}

(c) 2005 Elsevier B.V. All rights reserved.

Keywords: Classical mechanics; Dissipation; Positional forces; Instabilities

\section{Introduction}

Although the term was coined just recently by Bloch et al. [1,2], the counter-intuitive concept of a dissipationinduced instability has its origins in the classical work of Thomson (Lord Kelvin) and Tait [3] in 1879. Its theoretical basis was not properly understood until the 1950s in the works of Chetayev [4], Merkin [5] and other researchers. However, as the growing number of recent physical examples and applications demonstrates, these classical results are not appreciated and new physical examples are sometimes explained by rediscovering the theory. At the same time, as we shall see, the classical unified understanding of this apparently universal behavior allows one to link various phenomena and to find common features.

We consider here a generic canonical form of a finitedimensional system of second order equations

\footnotetext{
* Corresponding author. Tel.: +1 626395 3371; fax: +1 6267968914 .

E-mail address: rkrechet@cds.caltech.edu (R. Krechetnikov).
}

$\ddot{\mathbf{q}}+\underset{\text { gyro. }}{S \dot{\mathbf{q}}}+\underset{\text { dissip. }}{D \dot{\mathbf{q}}}+\underset{\text { pot. }}{C_{0} \mathbf{q}}+\underset{\text { positional }}{P \mathbf{q}}=\mathbf{N}$,

where the matrices $D, C_{0}$ are symmetric, $S, P$ are skewsymmetric, and $\mathbf{N}$ is a nonlinear term. In addition, $C_{0}$ is a diagonal matrix with each element of diag $C_{0}=$ $\left\{\lambda_{1}, \ldots, \lambda_{n}\right\}$ called, following terminology of Poincaré, a stability coefficient. This canonical form (1) has associated kinetic $T$ and potential $\Pi$ energies given by the quadratic forms $T=\frac{1}{2} \sum_{i} \dot{q}_{i}^{2}, \quad \Pi=\frac{1}{2} \sum_{i} \lambda_{i} q_{i}^{2}$.

The number of negative $\lambda_{i} \mathrm{~s}$ is called the degree of instability.

We are primarily concerned with instability phenomena, so we shall focus on the linear part of (1). The results retain their validity in the nonlinear case based on Lyapunov's theorem on stability which asserts nonlinear instability when one has spectral instability of the linearization. The canonical representation (1) is usually the result of a transformation of an original formulation; for a standard mechanics problem, such a transformation exists if both the mass matrix $a_{i j}$ 
and potential forces matrix are symmetric and the former is also positive definite. The original formulation could be the Euler-Lagrange equations with forcing for $n$ independent generalized coordinates,

$\mathrm{d}_{t} T_{\dot{q}_{i}}-T_{q_{i}}=Q_{i}, \quad Q_{i}=-\Pi_{q_{i}}+\widetilde{Q}_{i}$,

where $T=\frac{1}{2} a_{i k} \dot{q}_{i} \dot{q}_{k}$ is the kinetic energy and $Q_{i}$ are the generalized forces decomposed into potential and non-potential $\widetilde{Q}_{i}$ parts. Since the energy is given by $H=T+\Pi$, and its time evolution is

$\mathrm{d}_{t} H=\widetilde{Q}_{i} \dot{q}_{i}+\Pi_{t}$,

then, following Thomson and Tait [3], one can further classify the non-potential forces into gyroscopic, $\widetilde{Q}_{i} \dot{q}_{i}=0$, dissipative, $\widetilde{Q}_{i} \dot{q}_{i} \leq 0$, and accelerating, $\widetilde{Q}_{i} \dot{q}_{i} \geq 0$, forces. While these definitions are valid in the general nonlinear case, the corresponding terms in the linear parts in (1) highlight that the linear gyroscopic forces have a skew-symmetric structure $\widetilde{Q}_{i}=\gamma_{i k} \dot{q}_{k}, \gamma_{i k}=-\gamma_{k i}$, versus the dissipative forces, which have a symmetric form $\widetilde{Q}_{i}=-d_{i k} \dot{q}_{k}, d_{i k}=d_{k i}$. Physically, the dissipative forces could, for example, be due to motion in a resisting medium, etc., when the resistance depends only on the speed of motion. A special kind of force, not evident from the above classification, are the so-called positional forces which, similar to dissipative forces, change the energy of the system, but depend on the coordinates only, $\widetilde{Q}_{i}=-p_{i k} q_{k}, p_{i k}=-p_{k i}$. Such forces are typified by follower-forces.

The preceding classification of linear forces is important in studying the linear stability of various systems and allows one to identify the nature of various terms in the linearized dynamics. For a more complete classification of forces, including the nonlinear case which is beyond the scope of this introduction, we refer the reader to reviews by Merkin [6] and by Karapetyan and Rumyantsev [7].

The instabilities we study here are all mainly due to infinitesimal amounts of non-conservative effects in (1), thus making the conclusions generically applicable. The amplitude of non-conservative forces, though not important for the appearance of instabilities, defines the characteristic time of their evolution and thus are important in applications.

In Section 2 we state the two main results of the classical theory on the effect of non-conservative dissipative and positional forces relevant to our discussion and provide their geometrical interpretation. Section 3 is devoted to an interpretation of one of the recent examples in the literatureradiation-induced instability [8]—in the framework of the classical theory. In Section 4 we discuss the problem of objects levitating in a magnetic field, and explore the presence of both types of non-conservative forces as a generic phenomenon. Section 5 introduces the concept of a secondary dissipationinduced instability as coming from the interaction of both non-conservative effects. Finally, in Section 6 we discuss the challenge of extending these ideas for non-conservative effects to the case of infinite-dimensional systems such as to fluids, plasmas, and electromagnetism.

\section{Geometry of the classical theory}

The stability of conservative finite-dimensional systems is usually understood in the context of the Lagrange-Dirichlet theorem; that is, connected to the behavior of the second variation of the Hamiltonian. This also suggests a geometrical picture for the general non-conservative case and is the basic idea of Lyapunov's direct method, i.e. the construction of a function majorizing the dynamics.

Even though the system (1) is non-conservative, with the following definition of the Hamiltonian

$H=\frac{1}{2} \mathbf{p}^{T} I \mathbf{p}+\frac{1}{2} \mathbf{q}^{T} C_{0} \mathbf{q}, \quad \mathbf{p}=\dot{\mathbf{q}}$,

the system can be recast into a symplectic-metriplectic form. With $\mathbf{z}=(\mathbf{q}, \mathbf{p})$, the Eq. (1) can be written as

$\mathrm{d}_{t} \mathbf{z}=(J+G) H_{\mathbf{z}}$,

where the operators $J$ and $G$ are given by

$J=\left[\begin{array}{cc}0 & I \\ -I & -S\end{array}\right], \quad G=\left[\begin{array}{cc}0 & 0 \\ -P C_{0}^{-1} & -D\end{array}\right]$,

where the matrix $J$ is skew-symmetric and is called a Poisson operator, while the matrix $G$ in the absence of non-conservative positional forces is symmetric and called a metriplectic operator (in view of its similarity to a metric tensor). In the presence of non-conservative positional forces, the matrix $G$ is neither symmetric nor skew-symmetric. One can regard $J$ and $G$ as determining the geometry of phase space. The operator $J$ comes from symplectic geometry and is (1) non-singular, (2) skew-symmetric, and (3) obeys the Jacobi relation. If the operator $G$ is symmetric, i.e. contains only non-conservative dissipative forces, then its addition modifies the symplectic nature of phase space. The simplecticity is also destroyed by an addition of non-conservative positional forces.

Note that (2) is a valid Hamiltonian even if the gyroscopic forces are present (though in this case it corresponds to a noncanonical Hamiltonian form) and the effect of the gyroscopic forces appears through the non-canonical Poisson bracket (in which a sum on repeated indices is understood):

$\{F, H\}=F_{q_{i}} H_{p_{i}}-F_{p_{i}} H_{q_{i}}-S_{i j} F_{p_{i}} H_{p_{j}}$.

Gyroscopic forces can provide an exchange of energy among the modes, and thus can significantly alter the behavior of the system; for example, they can stabilize a non-zero degree of instability equilibrium.

The fundamental classical stability theorems [3-5], to be discussed here, can be deduced from two generic forms of the dynamical system (1) by appealing to spectral properties and by appealing to the geometrical properties as given by (3), respectively. In particular, a linear stability analysis of (3) amounts to the eigenvalue analysis of $(J+G) H_{\mathbf{z z}}$; in this context, one speaks of spectral stability or instability. Of course, spectral instability implies Lyapunov instability, but spectral stability need not imply Lyapunov stability.

Before going into the discussion of non-conservative effects, we first remember the main classical results for Hamiltonian 


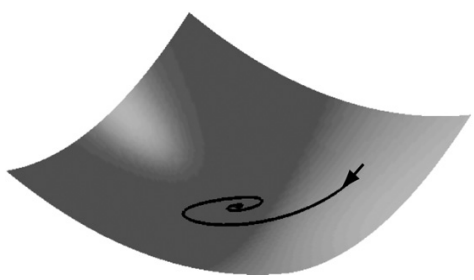

Fig. 1. System with a stable potential.

systems. The Lyapunov stability in this case is often inferred from the second variation of the Hamiltonian, which in the finite-dimensional case is the matrix (Hessian) of the second derivatives $H_{z_{i} z_{j}}$. This theorem for a finite dimensional system, known as the Lagrange-Dirichlet principle (1846), states that if the second variation of $H$ is definite at the equilibrium point, then the equilibrium point is Lyapunov stable. Definiteness of the second variation of $H$ is not necessary for stability, as noted by Cherry [9].

In its simplest form (Lagrange, 1788), this theorem applies to the case in which the Hamiltonian is separable and canonical (also called a simple mechanical system), i.e. if $H$ has the form $H=\frac{1}{2} \mathbf{p}^{T} I \mathbf{p}+V(\mathbf{q})$. In this case, there is an obvious connection between definiteness of $H(\mathbf{q}, \mathbf{p})$ and $V(\mathbf{q})$, as depicted in Figs. 1 and 2. In particular, an equilibrium point $\left(\mathbf{q}_{e}, \mathbf{p}_{e}=0\right)$ is stable if the second variation of $V$ at $\mathbf{q}_{e}$ is positive definite, i.e. if $\mathbf{q}_{e}$ is a strict local minimum of $V(\mathbf{q})$. This condition is sufficient but not necessary, as was first shown by Painlevé [10, 11]; that is, the converse (namely, instability in the absence of a minimum) to the Lagrange criterion is not true. However, additional conditions (apart from the absence of a minimum of $V(\mathbf{q}))$ allow one to formulate the converse to the Lagrange criterion; see [12] and references therein for more information. In particular, if the second variation is non-degenerate but indefinite, then one has spectral and hence Lyapunov instability.

As an illustration of the Lagrange-Dirichlet principle, we refer to Fig. 1, where the trajectory of a stable two-dimensional system

$\ddot{z}_{1}+d \dot{z}_{1}+c_{1} z_{1}=0$

$\ddot{z}_{2}+d \dot{z}_{2}+c_{2} z_{2}=0$,

with $d, c_{i}>0$, is projected onto the potential energy surface, $V\left(z_{1}, z_{2}\right)=\frac{1}{2} c_{i} z_{i}^{2}$ (again, a summation over $i$ is assumed). This type of geometric picture turns out to be very helpful in analyzing dissipation-induced instabilities.

Here we will discuss only one theorem by Thomson, Tait and Chetaev and one theorem by Merkin, which are directly pertinent to dissipation-induced instabilities. Instability in this context is understood to be spectral instability, which in turn implies Lyapunov instability of both the nonlinear system (1) and its linearization.

Theorem 1 (Thomson-Tait-Chetayev). If the system $\ddot{\mathbf{q}}+C_{0} \mathbf{q}=$ 0 has non-zero degree of instability, then (1) remains unstable after the addition of gyroscopic and dissipative forces with complete dissipation (that is, $\widetilde{Q}_{i} \dot{q}_{i}$ is negative definite).

This theorem implies that if a non-zero degree of instability equilibrium is stabilized with gyroscopic forces as in Fig. 2(a), then the stability is destroyed by an introduction of arbitrarily small dissipative forces. This result is illustrated in Fig. 2(c) for the following system:

$\ddot{z}_{1}+g \dot{z}_{2}+d \dot{z}_{1}+c_{1} z_{1}=0$

$\ddot{z}_{2}-g \dot{z}_{1}+d \dot{z}_{2}+c_{2} z_{2}=0$,

which has the equilibrium $(\mathbf{z}, \dot{\mathbf{z}})=(\mathbf{0}, \mathbf{0})$. If $c_{i}<0, i=1,2$, it has even degree of instability equal to 2 . This equilibrium point can be stabilized in the absence of dissipation, $d=0$, by adding gyroscopic forces provided that $|g|>\sqrt{-c_{1}}+\sqrt{-c_{2}}$.

The dynamics of this example can be interpreted, for instance, using the basic theory of the gyroscope, as that of the linearized equations of a Lagrange top, which is familiar to those who have spun a ball on their fingertip. Even when the top is deflected from the unstable (vertical) equilibrium position and is thus under action of destabilizing forces, a fast enough rotation makes it move in the direction perpendicular to the destabilizing force and to precess.

However, if the degree of instability is odd, as in Fig. 2(b), then the mechanism described above for gyroscopic stabilization does not work-gyroscopic stabilization is prohibited by another theorem of Thomson-Tait-Chetaev (see [3,4] and [1] for an exposition). Having the Lagrange top in mind, if the potential surface is as in Fig. 2(b), then the gyroscopic force changes its direction, passing from the concave to the convex part of the potential function, and thus violates conservation of angular momentum about the vertical axis. The addition of a dissipative force, $d>0$, destabilizes the system, regardless of its stability under the action of gyroscopic forces.

The counterpart of Theorem 1 for non-conservative positional forces is the following.

Theorem 2 (Merkin). The introduction of non-conservative positional forces (that is, the skew-symmetric matrix $P$ in (1) is non-zero) into a stable purely potential system, $\ddot{\mathbf{q}}+C_{0} \mathbf{q}=0$, with equal frequencies destroys the stability regardless of the form of the nonlinear terms.

For the history and other important results, we refer to Merkin [5,6], Zajac [13], and Agafonov [14]. To illustrate Theorem 2, consider the following system:

$\ddot{z}_{1}+p z_{2}+c z_{1}=0$,
$\ddot{z}_{2}-p z_{1}+c z_{2}=0$.

A study of the corresponding characteristic equation shows that the addition of non-zero, non-conservative, positional forces (that is, $p \neq 0$ ) to a stable system (that is, with degree of instability equal to zero) with equal frequencies makes it unstable, as shown in Fig. 2(d). Note that the second variation of the Hamiltonian of the original system is positive definite at the origin. This theorem applies to the analysis of linearized dynamics of isolated physical systems (such as the rotating shaft problem [15]) and of open physical systems (such as an elastic bar with a follower force). In the first case, this amounts to a route to dissipation-induced instabilities that is an alternative to the one given by the Thomson-Tait-Chetayev 


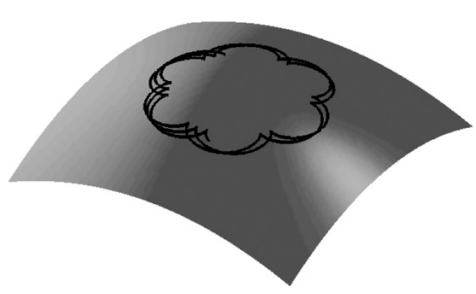

(a) Gyroscopic stabilization: even degree of instability.

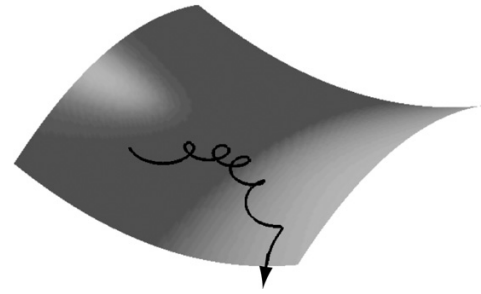

(b) Gyroscopic failure: odd degree of instability.

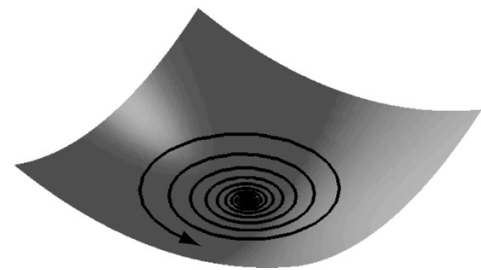

(d) Non-conservative positional destabilization of a system with stable potential.

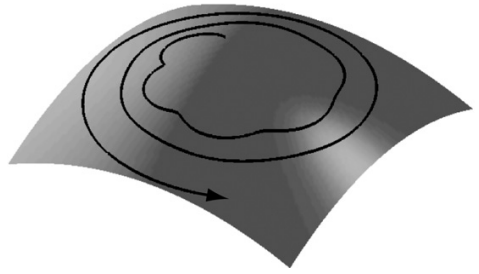

(c) Dissipative destabilization of a gyroscopically stable system.

Fig. 2. Projection of dynamics onto potential energy surface.

Theorem 1. Also, even though the theorem is formulated for the resonant case, it does lead to hyperbolic eigenvalues, so non-symmetric perturbations, which are small compared to the magnitude of the positional forces, do not destroy this instability behavior.

In conclusion, the basic physical effects of non-conservative (dissipative and positional) forces can be summarized as follows. If a stable equilibrium is formed from an unstable potential energy together with stabilizing gyroscopic forces, then this stability is destroyed by arbitrary dissipative forces. In contrast, if the stable equilibrium is formed from stable potential forces with equal frequencies alone, then the stability is destroyed by arbitrary non-conservative positional forces. It is notable that these two cases both have antisymmetric coupling in system (1), which basically prohibits the construction of a Lyapunov function to prove stability. The instabilities occur in both cases due to the breaking of symmetry in the original conservative system (and its phase space) so that the eigenvalues move away from the imaginary axis.

\section{Radiation-induced instability}

As an application of the Thomson-Tait-Chetayev result (Theorem 1), we consider the work [8], in which the phenomenon of radiation-induced instability is developed. Here we show that this instability can be understood by using Theorem 1.

The physical set-up is modeled by a finite-dimensional system coupled to an infinite-dimensional system that is responsible for a process of wave radiation. For the development and history of this model, we refer to Soffer and
Weinstein [16]. The resulting governing system has the form

$$
\begin{aligned}
& \ddot{x}+g \dot{y}+\alpha x=\gamma \int_{0}^{t}[x(s)+y(s)] \mathrm{d} s, \\
& \ddot{y}-g \dot{x}+\beta y=\gamma \int_{0}^{t}[x(s)+y(s)] \mathrm{d} s,
\end{aligned}
$$

where the right hand side describes the effects of radiation. The work [8] establishes the Lyapunov instability of this system as being caused by the presence of radiation even when the mechanical part (i.e. the left-hand side) of (6) is spectrally stable, say gyroscopically stabilized.

The proof in [8] is based on differentiation of the above system followed by a direct analysis. On the other hand, in our approach, we introduce another variable, $z=\int_{0}^{t}[x(s)+y(s)] \mathrm{d} s$, so that the preceding system reads

$\ddot{\phi}+G \dot{\phi}+C \phi=\mathbf{0}, \quad \phi=[x, y, z]^{T}$,

where $G$ and $C$ are defined by

$G=\left[\begin{array}{ccc}0 & -g & 0 \\ g & 0 & 0 \\ -1 & -1 & 0\end{array}\right], \quad C=\left[\begin{array}{ccc}\alpha & 0 & -\gamma \\ 0 & \beta & -\gamma \\ 0 & 0 & 0\end{array}\right]$.

Introducing the change of variables, $\phi^{\prime}=A \phi$, where $A$ is such that $A C A^{-1}$ is diagonal, we arrive at

$\ddot{\phi}^{\prime}+\widetilde{G} \dot{\phi}^{\prime}+\widetilde{C} \phi^{\prime}=\mathbf{0}, \quad \widetilde{C}=\left[\begin{array}{ccc}\alpha & 0 & 0 \\ 0 & \beta & 0 \\ 0 & 0 & 0\end{array}\right]$,

where

$\widetilde{G}=\left[\begin{array}{ccc}\frac{\gamma}{\alpha} & \frac{\gamma}{\alpha}-g & \frac{\gamma^{2}}{\alpha^{2}}+\frac{\gamma}{\beta}\left(\frac{\gamma}{\alpha}-g\right) \\ \frac{\gamma}{\beta}+g & \frac{\gamma}{\beta} & \frac{\gamma^{2}}{\beta^{2}}+\frac{\gamma}{\alpha}\left(\frac{\gamma}{\beta}+g\right) \\ -1 & -1 & -\frac{\gamma}{\alpha}-\frac{\gamma}{\beta}\end{array}\right]$, 


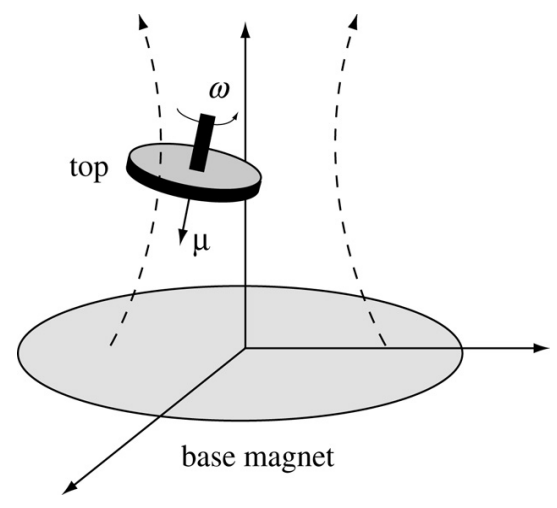

Fig. 3. Schematics of Levitron.

where the new matrix $\widetilde{G}$ has non-degenerate symmetric (dissipative) and anti-symmetric (gyroscopic) parts. Therefore, if the system with $\widetilde{G}=0$ is unstable, then adding arbitrary gyroscopic and dissipative forces leaves it unstable, in accordance with Theorem $1 .^{1}$

\section{The Levitron}

The invention of a levitating magnetic object by Harrigan [17] overcame the taboo imposed by the Earnshaw theorem [18] and received some resonance in the literature [19,20]. However, a closer look at this problem reveals that Harrigan had very strong intuitive reasons that are, in fact, supported by the Thomson-Tait-Chetayev theory. As discussed in Section 2, this theory predicts the possibility of gyroscopic stabilization of an unstable system (with non-zero degree of instability), a fact that is used in numerous engineering applications (such as a monorail car, etc.). It is curious that, despite this theory, the explanation of stabilization in the literature was based on a more modern but approximate theory based on adiabatic invariants (cf. Berry [19] and Simon et al. [20]).

The dynamics of a point magnetic dipole of strength $\mu$ and mass $M$ in an axisymmetric magnetic field $\mathbf{B}$, as shown in Fig. 3, are governed by torque and force balance as follows:

$\mathrm{d}_{t} \boldsymbol{\mu}=\frac{\mu}{I \omega} \boldsymbol{\mu} \times \mathbf{B}$,
$M \mathrm{~d}_{t}^{2} \mathbf{r}=\nabla(\boldsymbol{\mu} \cdot \mathbf{B})-M g \widehat{z}$.

The magnetic field can be represented in the neighborhood of its axis of symmetry by means of a Taylor series expansion:

$$
\begin{aligned}
& B_{z}=B_{0}+S z+K z^{2}-\frac{1}{2} K r^{2}+\cdots, \\
& B_{r}=-\frac{1}{2} S r-K r z+\cdots .
\end{aligned}
$$

With the following non-dimensionalization,

$\boldsymbol{\mu} \rightarrow a \boldsymbol{\mu}, \mathbf{r} \rightarrow \alpha \mathbf{r}, t \rightarrow \gamma t ; \quad \frac{a}{\alpha}=\frac{\omega_{p}}{\omega} \frac{\mu^{2} M}{I}$,

\footnotetext{
${ }^{1}$ Note that, even though the classical Thomson-Tait-Chetayev theory was developed for the non-critical case (i.e. all the stability coefficients are nonzero), its physical implications are wider, and in many situations, including this one, an examination of the proofs shows that the theorems are still true and yield correct predictions.
}

$\gamma=\sqrt{\frac{M}{I \omega}}, \quad \omega_{1}=\gamma \frac{a}{\alpha} \frac{S}{2 M}, \quad \omega_{2}=\gamma \omega_{p}$

the linearized equations written in a component form become:

$$
\begin{gathered}
\left(\frac{\mathrm{d}^{2}}{\mathrm{~d} t^{2}}-\left[\begin{array}{cccc}
0 & 0 & 0 & -\frac{\omega_{1}}{\omega_{2}} \\
0 & 0 & \frac{\omega_{1}}{\omega_{2}} & 0 \\
0 & -\frac{\omega_{1}}{\omega_{2}} & 0 & 0 \\
\frac{\omega_{1}}{\omega_{2}} & 0 & 0 & 0
\end{array}\right] \mathrm{d}\right. \\
- \\
-\left[\begin{array}{cccc}
1+\frac{\omega_{1}^{2}}{\omega_{2}^{2}} & 0 & 0 & 0 \\
0 & 1+\frac{\omega_{1}^{2}}{\omega_{2}^{2}} & 0 & 0 \\
-\omega_{1} & 0 & -\omega_{2}^{2} & 0 \\
0 & -\omega_{1} & 0 & -\omega_{2}^{2}
\end{array}\right]\left[\begin{array}{c}
x \\
y \\
\mu_{x} \\
\mu_{y}
\end{array}\right]\left[\begin{array}{c}
x \\
y \\
\mu_{x} \\
\mu_{y}
\end{array}\right]=0 .
\end{gathered}
$$

It is obvious that the degree of instability of this system is even, so that one can expect stabilization for a certain ratio of frequencies $\omega_{1}$ and $\omega_{2}$, since the stabilization is achieved only at certain amplitude of gyroscopic force.

The dissipative effects in a spinning top are known to be crucial, since they determine the finite lifetime of a stable levitation [20]. However, those effects are, in general, very complicated due to the top's finite size, conductivity, magnetization, interaction with air, etc. Here we just discuss qualitatively the origin of various forces and moments and to make one key point: eddy currents introduce both types of nonconservative forces, i.e. dissipative and positional.

The actual Levitron is usually made of a rod attached to a ring or disk magnet. Here we assume, for simplicity, a toroidal shape of the top magnet, which is of ferromagnetic type and is conducting (being made of iron, or various iron alloys). The magnetic field is considered to be axisymmetric, but decaying when one moves away from the base magnet and away from the axis of symmetry. As is known from experimental observations, the top is not rotating with its axis exactly aligned with the axis of symmetry. Rather, it performs a precession and nutation motion. This implies that the center of the torus is off the axis of symmetry and the plane of the torus is performing oscillatory motion (wobbling) about the horizontal plane. Now, on the basis of Faraday's law of induction, the induced electromotive force equals the negative rate of change of flux:

$\mathcal{E}^{\text {ind }}=-\frac{1}{c} \frac{\mathrm{d} \Phi}{\mathrm{d} t}$,

which is the consequence of the Lorentz force law, $\mathbf{F}=\frac{e}{c} \mathbf{v} \times$ $\mathbf{H}$. This means that, if the top is only precessing, then the flux through the torus contour is constant, and thus there are no currents induced. However, nutation makes the flux timedependent and, if the magnet were just a linear conductor, the induced current would be only of the type shown in Fig. 4(a) and denoted $\mathbf{j}_{1}$. However, the complicated behavior of the top and its finite size induces eddy currents of sophisticated topology, which can be obtained by solving the complete time-dependent field equations, which is not really feasible. 


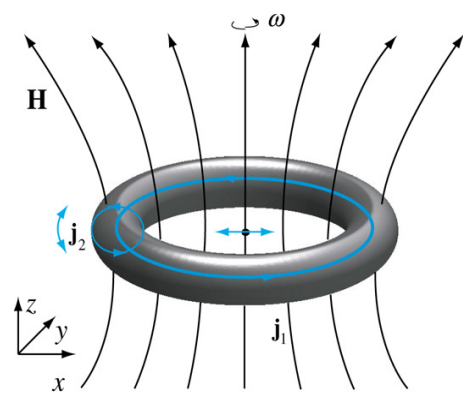

(a) On the origin of eddy currents.

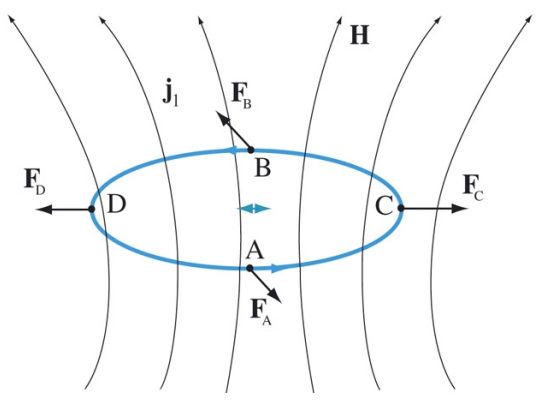

(b) Currents of $\mathbf{j}_{1}$-type.

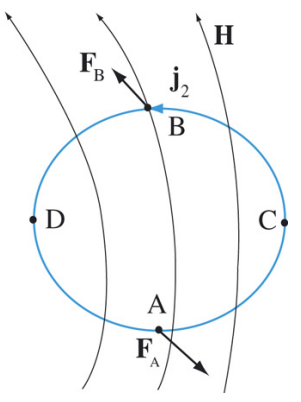

(c) Currents of $\mathbf{j}_{2}$-type.

Fig. 4. Forces on induced currents.

However, in view of the torus geometry of the top, one can decompose the general elementary current $\mathbf{j}$ into the currents of two types, $\mathbf{j}_{1}$ and $\mathbf{j}_{2}$, as shown in Fig. 4(a).

Once the origin of the currents is clarified, we can develop an understanding of the forces acting on the top magnet besides the interaction of the external magnetic field and the top magnetic moment. The general formula tells us that

$\mathbf{F}=\frac{I}{c} \oint \mathbf{d l} \times \mathbf{H}$.

In the case of the elementary current $\mathbf{j}_{1}$, the torus is displaced to the left along the $x$-axis (cf. Fig. 4(b)) and thus the point $\mathrm{C}$ experiences a stronger magnetic field than the point $\mathrm{D}$. The configuration is symmetric about the $x$-axis, so we do not need to take into account the force balance between points $\mathrm{A}$ and $\mathrm{B}$. For a given configuration of current and magnetic field direction, the resulting force is restoring. Such a force arises when one moves the torus away from axis of symmetry, since the induced current resists the imposed motion. Also, the induced current has its own magnetic moment that interacts with the external magnetic field and contributes to the angular momentum equations. However, in general the force resulting from the presence of $\mathbf{j}_{1}$ might be pushing the top away from the axis of symmetry. In the case of the elementary current $\mathbf{j}_{2}$, the forces at points $C$ and $D$ vanish, so we need to analyze the forces at the points $\mathrm{A}$ and $\mathrm{B}$, which are closer to and farther from a base magnet, respectively. The torus is again displaced along the $x$-axis, but, as one can see, the fact that the magnetic field at A is stronger than at B leads to a resulting force in the $y$-direction, along with an overturning moment. An analogous skew-symmetry arises in the rotating shaft problem [15] and leads to destabilization due to positional forces. In general, one can expect the forces to be of all these types. Regular dissipation arises due to friction with air and due to the resistance of the conductor. If the latter is $R$, then the induced total current is $I=\mathcal{E}^{\text {ind }} / R$ and the eddy current induced losses are $I^{2} R$. Since the origin of the fluctuating magnetic flux is through a nutation motion, for example, then the underlying dissipative force should be proportional to the velocity components. The presence of both types of non-conservative forces implies that the Levitron will always be unstable (though the characteristic time of instability can be large) unless these dissipative effects are compensated with external pumping of energy, as is often done in gyroscopic systems [5].
In concluding this section, we should mention that the simultaneous appearance of both types of non-conservative forces is very common in real world systems. One of many examples is discussed in the next section. Another famous example is the instability of shafts rotating in hydrodynamic media treated by Kapitsa [15], who assumed that friction is proportional to squared relative velocity and, after integration of these forces over the shaft's perimeter, obtained the resultant non-conservative forces of pure positional type. However, in reality the friction law is a general function of velocity and other variables, so that one can expect the presence of dissipative forces as well.

\section{Secondary instability}

In this section we continue the discussion of the simultaneous appearance of both types of non-conservative effects and demonstrate their combined effect, which leads to the notion of a secondary dissipation-induced instability phenomenon; this phenomenon was discovered by Ziegler [21] in the context of elastic systems.

We consider a system of this type, namely two identical bars of length $l$ and mass $m$, and torsional springs of stiffness $c_{0}$, as shown in Fig. 5. For simplicity, the two-bar system is restricted to a plane and not subjected to a gravity field. The moment of inertia of the first bar with respect to the point of attachment $\mathrm{O}$ is $J_{1}$, and that of the second bar with respect to its center of mass is $J_{2}$. With these definitions, the kinetic and potential energies of the system for small deflections $\phi_{1,2}$, that is, for the linearization of the system, are

$T=\frac{1}{2}\left(a_{11} \dot{\varphi}_{1}^{2}+2 a_{12} \dot{\varphi}_{1} \dot{\varphi}_{2}+a_{22} \dot{\varphi}_{2}^{2}\right)$,

$\Pi=\frac{1}{2} c \varphi_{1}^{2}+\frac{1}{2} c\left(\varphi_{2}-\varphi_{1}\right)^{2}$,

where $a_{11}=J_{1}+m l^{2}, a_{12}=\frac{1}{2} m l^{2}, a_{22}=J_{2}+\frac{1}{4} m l^{2}$. The resulting Euler-Lagrange equations for the angles $\varphi_{1}$ and $\varphi_{2}$ are:

$\left[\begin{array}{ll}a_{11} & a_{12} \\ a_{12} & a_{22}\end{array}\right]\left[\begin{array}{l}\ddot{\varphi}_{1} \\ \ddot{\varphi}_{2}\end{array}\right]+\left(\left[\begin{array}{ll}c_{11} & c_{12} \\ c_{12} & c_{22}\end{array}\right]+\left[\begin{array}{cc}0 & p \\ -p & 0\end{array}\right]\right)\left[\begin{array}{l}\varphi_{1} \\ \varphi_{2}\end{array}\right]=0$,

where $a_{11}=\frac{4}{3} m l^{2}, a_{12}=\frac{1}{2} m l^{2}$, and $a_{22}=\frac{1}{3} m l^{2} ; c_{11}=$ $2 c_{0}-F l, c_{12}=\frac{1}{2} F l-c_{0}, c_{22}=c_{0}$, and $p=\frac{1}{2} F l$. The system 


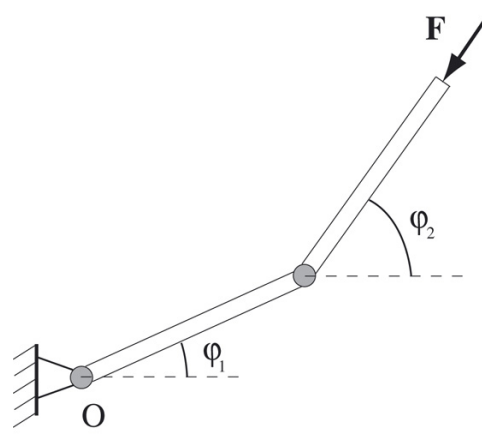

Fig. 5. Schematics of cantilever-elastic bar.

(8) can be reduced to the form (1) using the standard technique for diagonalization of pencils of matrices (or quadratic forms), as in [22]. Note that the follower force contributes to both the positional $P$ and potential $C$ matrices, and thus the situation is slightly more general than the one treated by Merkin's theorem. An eigenvalue analysis of Eq. (8) leads to a quartic equation for $\lambda^{2}$ of the form $a \lambda^{4}+b \lambda^{2}+c=0$; this shows that the solution is stable if $\lambda^{2}<0$, that is

$b>0, \quad b^{2}-4 a c>0$,

which yields $F<\frac{6}{5}\left(3-\sqrt{\frac{20}{3}}\right) \frac{c_{0}}{l}$. The stability breaks when the magnitude of the follower force exceeds this value, and thus the second inequality in (9) changes its sign. This model was studied by Nikolai [23] as an approximation for the effects occurring due to the outflow of combustion gases in jet engines. The same model, in a finite-dimensional approximation, is applicable to a continuous cantilever conveying liquid [24].

In addition to the positional (follower) force $F$, there are regular dissipative forces, so one can introduce a regular dissipation term into the previous example (8) and study the effect of two non-conservative forces (1), namely the matrix $P$ (positional) and regular dissipation $D$. The origin of $D$ can be due, for instance, to hydrodynamic friction inside the bar-tubes through which there is a flow of liquid, and the ejection of which creates a follower force similar to that in a jet engine. Suppose for simplicity that the dissipation matrix $D$ is diagonal with equal diagonal entries of magnitude $\epsilon$, and that the magnitude of the follower force is slightly below its critical value; that is, the system is close to buckling.

If we perform an asymptotic study of eigenvalues, and write $\lambda=\lambda_{0}+\epsilon \lambda_{1}$, where $\lambda_{0}$ is the eigenvalue of the problem without dissipation (the stable configuration), then it is straightforward to show that

$\lambda_{1}=-\frac{c_{0}+e_{1}+\left(a_{11}+a_{22}\right) \lambda_{0}^{2}}{2\left(b+2 a \lambda_{0}^{2}\right)}, \quad e_{1}=2 c_{0}-F l ;$

that is, under assumptions of stability of the non-dissipative system (9) and in the case when both $a>0$ and $c>0$ (one can show that this is physically realizable), the system experiences instability, $\lambda_{1}>0$, for an arbitrary small dissipation $\epsilon$.

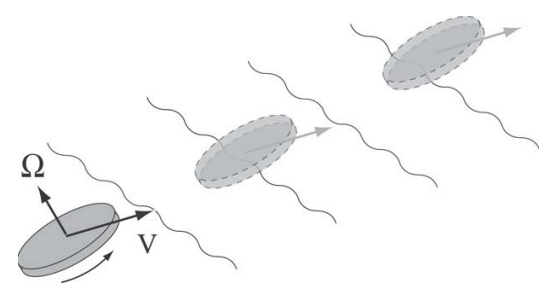

Fig. 6. Skipping stone.

\section{Discussion}

In this work we have illustrated the power of two classical theorems due to Thomson-Tait-Chetayev and Merkin concerning the destabilizing non-conservative effects. This was shown by analyzing several phenomena currently under discussion in the literature (such as radiation-induced instabilities, the Levitron, etc.) using these classical results.

We have also introduced a basic geometric interpretation of the two fundamental non-conservative destabilization mechanisms (dissipation that destabilizes a gyroscopically stabilized system and positional forces that can destabilize a system even at an energy minimum, as shown in Fig. 2(c) and (d)) and demonstrated that one can have the simultaneous appearance of both types of non-conservative effects in physical systems. The behavior of the second variation $\delta^{2} H$ at the equilibrium point selects the appropriate type of destabilizing non-conservative forces. Also, the interaction of both types of non-conservative effects naturally leads to the notion of secondary dissipation-induced instabilities.

As the structure of the complete fundamental system (1) and the associated geometric picture in Fig. 2 suggest, the two non-conservative destabilizing effects that we have discussed exhaust the most fundamental possibilities for linear instabilities in finite-dimensional mechanical systems.

At this stage there is no analogous rigorous theory for infinite-dimensional systems, and a naive translation of the above results can lead to a number of pitfalls associated with infinite dimensionality, as shown in [25] and [26]. However, the abundance of many infinite-dimensional physical systems, such as fluid systems, for which this sort of theory is formally applicable, requires further developments. The discussion in Section 4 on the Levitron problem already indicates that, for some systems, an appropriate description involves the coupling of rigid body and field dynamics.

Another fascinating physical system-skipping stones (cf. Fig. 6) - also exhibits the coupling of rigid body and field equations. However, besides the oversimplified phenomenological theory [27], there is no adequate (even approximate finite-dimensional) description of the skipping stone problem, which would allow one to understand the physics better. It is notable that skipping stones (cf. Fig. 6) require an initial spin $\Omega$ for gyroscopic stabilization [27] and their interaction with the underlying fluid leads to dissipation. Therefore, an approximate, finite-dimensional, description should fit the universal picture introduced in the present paper. In particular, the known fact of gyroscopic stabilization and the presence of dissipation in this problem should allow one 
to conclude that the skipping stone will always be unstable, in accordance with experience and with Theorem 1; as in the Levitron, depending on the details of the particular situation, the characteristic time of instability can be large.

Finally, we note that there are also infinite-dimensional systems which do not readily admit an approximate finitedimensional mechanical analog. This class of essentially infinite-dimensional problems usually corresponds to field equations without coupling to rigid body dynamics. One such example is the baroclinic instability - a large scale instability of the westerly winds in mid-latitudes (see [25]). It is curious that the destabilizing Eckman layer dissipation in this problem corresponds to the geometrical picture 2(d), i.e. one has a stable equilibrium with definite second variation $\delta^{2} H$ that is destabilized by non-conservative forces that are analogous to positional forces in the finite-dimensional case.

\section{Acknowledgements}

This research was partially supported by NSF-ITR Grant ACI-0204932. The authors also thank Nawaf Bou-Rabee for bringing his work [24] to our attention and for a stimulating discussion.

\section{References}

[1] A. Bloch, P.S. Krishnaprasad, J.E. Marsden, T.S. Ratiu, Dissipation induced instabilities, Ann. Inst. H. Poincaré Anal. Non Linéaire 11 (1994) 37-90.

[2] A. Bloch, P.S. Krishnaprasad, J.E. Marsden, T.S. Ratiu, The EulerPoincaré equations and double bracket dissipation, Comm. Math. Phys. 1975 (1996) 1-42.

[3] W. Thomson, P.G. Tait, Treatise on Natural Philosophy, Cambridge University Press, 1879.

[4] N.G. Chetayev, The Stability of Motion, Pergamon Press, 1961.

[5] D.R. Merkin, Introduction to the Theory of Stability, Springer-Verlag, 1997.

[6] D.R. Merkin, Gyroscopic Systems, Nauka, Moscow, 1974 (in Russian) (first edition, 1956).

[7] A.V. Karapetyan, V.V. Rumyantsev, Stability of conservative and dissipative systems, Itogi Nauki Tekh. 6 (1983) 3-128.
[8] P. Hagerty, A.M. Bloch, M.I. Weinstein, Radiation induced instability in interconnected systems, in: Proceedings of the 38th CDC, IEEE, 1999, pp. 651-656.

[9] T.M. Cherry, Some examples of trajectories defined by differential equations of a generalized dynamical type, Trans. Cambridge Philos. Soc. 23 (1925) 165-200.

[10] P. Painlevé, Sur la stabilité de l'équilibre, C. R. Acad. Sci. Paris 138 (1904) 1555-1557.

[11] A. Wintner, The Analytical Foundations of Celectial Mechanics, Princeton University Press, 1947.

[12] V.V. Rumyantsev, S.P. Sosnitskii, On the instability of the equilibrium of holonomic conservative systems, J. Appl. Math. Mech. 57 (1994) 1101-1122.

[13] E.E. Zajac, The Kelvin-Tait-Chetaev theorem and extensions, J. Astronaut. Sci. 11 (1964) 46-49.

[14] S.A. Agafonov, Stability and motion stabilization of nonconservative mechanical systems, J. Math. Sci. 112 (2002) 4419-4497.

[15] P.L. Kapitsa, Stability and transition through the critical speed of fast rotating shafts with friction, Zh. Tekh. Fiz. 9 (1939) 124-147.

[16] A. Soffer, M.I. Weinstein, Resonances, radiation damping and instability in Hamiltonian wave equations, Invent. Math. 136 (1999) 9-74.

[17] R.M. Harrigan, U.S. patent 4,382,245, 1983.

[18] S. Earnshaw, On the nature of the molecular forces which regulate the constitution of the luminiferous ether, Trans. Cambridge Philos. Soc. 7 (1842) 97-112.

[19] M.V. Berry, The levitron: an adiabatic trap for spins, Proc. R. Soc. A 452 (1996) 1207-1220.

[20] M.D. Simon, L.O. Heflinger, S.L. Ridgway, Spin stabilized magnetic levitation, Amer. J. Phys. 65 (1997) 286-292.

[21] H. Ziegler, Die Stabilitätskriterien der Elastomechanik, Ing.-Arch. 20 (1952) 49-56.

[22] F.R. Gantmacher, The Theory of Matrices, Chelsea Pub. Co., 1977.

[23] E.L. Nikolai, Theoretical Mechanics, GONTI, Moscow, 1939 (in Russian).

[24] N.M. Bou-Rabee, L.A. Romero, A.G. Sallinger, A multiparameter, numerical stability analysis of a standing cantilever conveying fluid, SIAM J. Appl. Dyn. Syst. 1 (2002) 190-214.

[25] R. Krechetnikov, J.E. Marsden, Dissipation-induced instability phenomena in infinite-dimensional systems, 2005 (submitted for publication).

[26] J.M. Ball, J.E. Marsden, Quasiconvexity at the boundary, positivity of the second variation and elastic stability, Arch. Ration. Mech. Anal. 86 (1984) 251-277.

[27] L. Bocquet, The physics of stone skipping, Amer. J. Phys. 71 (2003) 150-155. 